\title{
Sputtering of Thick Deuterium Films by KeV Electrons
}

\author{
Thestrup Nielsen, Birgitte; Svendsen, Winnie Edith; Schou, Jørgen; Ellegaard, Ole
}

Published in:

Physical Review Letters

Link to article, DOI:

10.1103/PhysRevLett.73.1444

Publication date:

1994

Document Version

Publisher's PDF, also known as Version of record

Link back to DTU Orbit

\section{Citation $(A P A)$ :}

Thestrup Nielsen, B., Svendsen, W. E., Schou, J., \& Ellegaard, O. (1994). Sputtering of Thick Deuterium Films by KeV Electrons. Physical Review Letters, 73(10), 1444-1447. https://doi.org/10.1103/PhysRevLett.73.1444

\section{General rights}

Copyright and moral rights for the publications made accessible in the public portal are retained by the authors and/or other copyright owners and it is a condition of accessing publications that users recognise and abide by the legal requirements associated with these rights.

- Users may download and print one copy of any publication from the public portal for the purpose of private study or research.

- You may not further distribute the material or use it for any profit-making activity or commercial gain

- You may freely distribute the URL identifying the publication in the public portal 


\title{
Sputtering of Thick Deuterium Films by keV Electrons
}

\author{
Birgitte Thestrup, Winnie Svendsen, Jørgen Schou, and Ole Ellegaard* \\ Department of Optics and Fluid Dynamics, Ris $\phi$ National Laboratory, DK-4000 Roskilde, Denmark
}

(Received 9 May 1994)

Sputtering of thick films of solid deuterium up to several $\mu \mathrm{m}$ by keV electrons is reported for the first time. The sputtering yield increases within a narrow range of thicknesses around $1.6 \mu \mathrm{m}$ by about 2 orders of magnitude for $1.5 \mathrm{keV}$ electrons. A similar behavior has not been observed for ion bombardment. The yield enhancement is accompanied by an increasing electron accumulation in the film.

PACS numbers: $79.20 . \mathrm{Kz}, 34.80 . \mathrm{Gs}, 72.20 . \mathrm{Jv}$

Surfaces of the solid hydrogen molecules are the most volatile targets that can be studied with a chargedparticle beam in a laboratory vacuum system [1,2]. The binding energy of the hydrogenic molecules to the lattice site is extremely low corresponding to a sublimation energy of about $10 \mathrm{meV} /$ molecule $[3,4]$. Therefore, beam-induced evaporation occurs frequently in any particle-beam experiment with these solids, unless the target temperature and the beam power are kept below a certain threshold $[1,2,5,6]$.

The hydrogenic solids are unique as well because of the extremely small charge mobility. Only the negative carriers have a measurable mobility [7]. These carriers are probably electrons in a polaronic state, in which the electron wave packet is spread over and bound to a small region of local distortion of the lattice [8]. A minor fraction becomes completely immobile after trapping in bubbles [9]. The electron generates a bubble in a preexisting defect of the lattice because of the pronounced zero-point motion of the electron [8]. These bubbles exist in tens of minutes after the ionizing beam has been turned off even at temperatures around $4.2 \mathrm{~K}[8,10]$. Until recently, any charge accumulation in these solids has not been associated systematically with sputtering.

Sputtering is characterized as the erosion of surfaces by individual particle impacts [5]. For keV hydrogen ion incidence on a hydrogenic surface sputtering takes place predominantly via electronic transitions. The incident particle generates excitations and ionizations along the path. Many of these excited states decay to repulsive branches so that the molecules immediately dissociate. The repulsing atoms may initiate collision cascades, and when molecules close to the surface acquire sufficient kinetic energy, they can pass the surface barrier and be emitted from the material $[1,2,5]$.

The sputtering experiments performed with hydrogen ion beams have not shown any major discrepancies with other volatile solids, even though the electronic transitions leading to sputtering are not yet identified [1,2,11]. For hydrogen ions a typical example is shown in Fig. 1(a); the yield reaches a saturation value which has been interpreted as a "bulk" yield [12]. This value has been used in Refs. [1,2]. The sputtering yields are very high, i.e., up to $1000 \mathrm{H}_{2} / \mathrm{H}$ at $10 \mathrm{keV}$ proton incidence, but this is essentially a consequence of the low binding energy of the molecules to the lattice. We found a strong dependence on the target isotope from the most volatile isotope, solid $\mathrm{H}_{2}$, up to the least volatile stable one, solid $\mathrm{D}_{2}$, as well [1].

Comprehensive sputtering experiments with electron beams have been carried out largely for solid $\mathrm{D}_{2}$

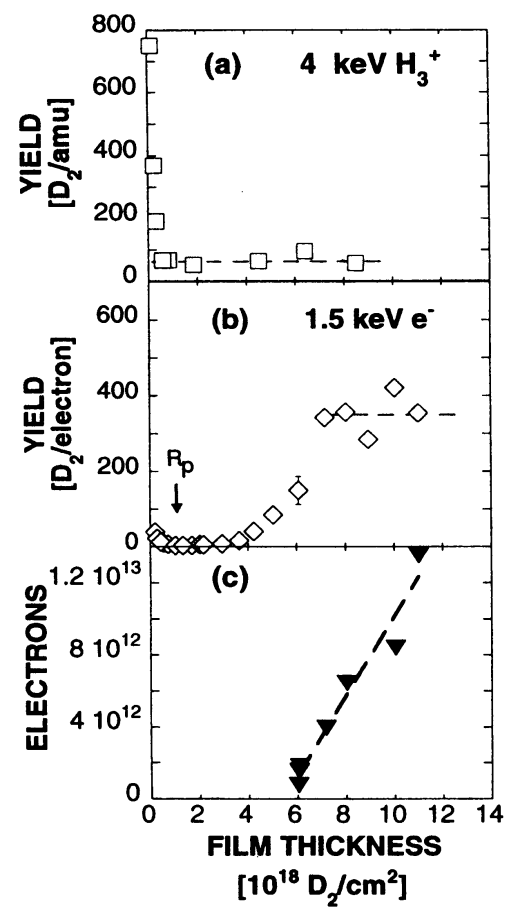

FIG. 1. (a) The sputtering yield as a function of initial thickness for $4 \mathrm{keV} \mathrm{H}_{3}{ }^{+}$ions from Ref. [12]; the dashed line indicates the bulk yield per projectile atom $\left[67.4 \mathrm{D}_{2} /(\mathrm{H}\right.$ atom $\left.)\right]$. (b) $1.5 \mathrm{keV}$ electrons; $R_{p}$ is the average projected range from Ref. [16]. A typical standard deviation is indicated. The dashed line shows the saturation yield $\left(352 \mathrm{D}_{2}\right.$ /electron). (c) Number of accumulated electrons in a thick film as a function of film thickness for $1.5 \mathrm{keV}$ electron bombardment. For each thickness the number is determined from the area similar to that in Fig. 3(b). 
$[6,11,13]$. The yield decreases from a high thin-film yield to a low thick-film yield value around film thicknesses that correspond to twice the average projected range for electrons. Since a considerable enhancement of the sputtering yield was seen for thicknesses larger than 3 times the range some years ago [11,14], we have explored this effect in a new series of measurements on very thick deuterium films. In this work we present results that show an enhancement of the sputtering yield by 2 orders of magnitude within a narrow range of film thicknesses. This surprising increase is accompanied by a pronounced charge accumulation in the thick films.

The experimental setup (Fig. 2) resembles that used for hydrogen ion bombardment of hydrogenic films described previously $[1,6,11]$. Films of solid $\mathrm{D}_{2}$ of a thickness from $0.2 \times 10^{18}$ up to $13 \times 10^{18} \mathrm{D}_{2} / \mathrm{cm}^{2}$ (corresponding to $4 \mu \mathrm{m}$ ) are produced by letting a jet of cooled gas impinge on an oscillating-quartz-crystal microbalance suspended below a liquid-helium cryostat. With this system it is possible to make $D_{2}$ films of known thickness and to measure the mass loss during the subsequent irradiation. Electron beams of 1.5 and $2 \mathrm{keV}$ are directed onto the target by a system of external voltage plates. In order to ensure a homogeneously irradiated area on the target, the beam is swept both horizontally and vertically over an aperture in front of the target. The beam current $I_{B}$ is measured before and after irradiation by deflecting the beam into a Faraday cup. We have applied a negatively biased $(-90 \mathrm{~V})$ repeller ring in front of the target to suppress the secondary electrons. This ring has turned out to reduce charge-up problems largely in our previous work $[1,6,11,15]$. During the measurements the target current $I_{T}$ as well as the frequency of the microbalance are recorded. Under normal circumstances, e.g., without charge-up as a result of electron or ion accumulation in the film, the difference between the target and beam current $\Delta=\left(I_{B}-I_{T}\right) / I_{B}$ accounts for the number of

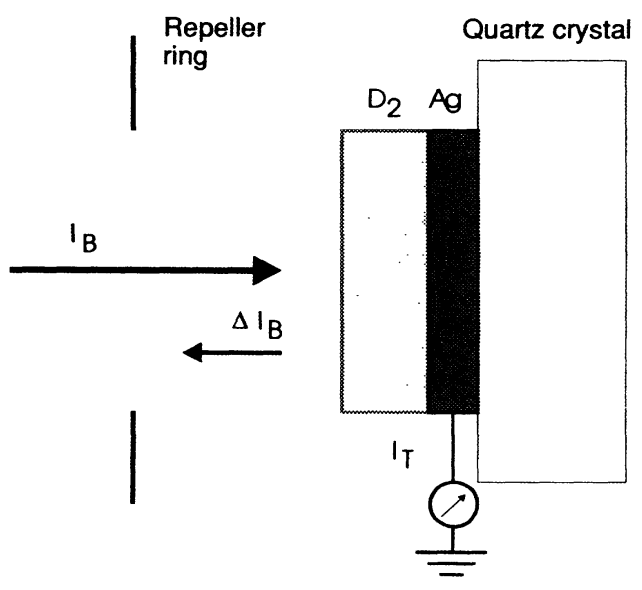

FIG. 2. Schematic view of the target area. The currents are shown as well. electrons reflected from the target. In that case $\Delta$ is equal to the reflection coefficient $\eta$ for the hydrogen isotopes (about 0.01 for $\mathrm{keV}$ electrons of normal incidence [16]).

The temperature of the pumped helium bath reached a value of $2.0 \mathrm{~K}$. The 4000 - $\AA$-thick silver electrode of the quartz crystal was thermally connected to the quartz-crystal holder and the cryostat bottom by a lowtemperature-solder point directly on the electrode. The precise temperature of the electrode cannot be measured because of the high-frequency oscillations.

The important test showing that sputtering rather than beam-induced evaporation takes place was performed in two independent ways. The yield obtained at film thicknesses comparable to the range agreed well with that obtained previously for $\mathrm{D}_{2}$ films deposited on a massive gold substrate [14]. At such film thicknesses it was possible to monitor the erosion - and in turn to determine the sputtering yield-from variations in the reflection coefficient $\eta[5,11]$. As a second check we measured the yield for different beam currents at a thickness around $6 \times 10^{18} \mathrm{D}_{2} / \mathrm{cm}^{2}$. The yield did not show any significant dependence on the current for values less than $160 \mathrm{nA}$. A typical current used was $100 \mathrm{nA}$.

The yield as a function of film thickness for $1.5 \mathrm{keV}$ electron bombardment is shown in Fig. 1(b). One notes the strong increase from the minimum of about $3.5 \mathrm{D}_{2}$ /electron up to the saturation value of $350 \mathrm{D}_{2} /$ electron for thicknesses that exceed $8 \times 10^{18} \mathrm{D}_{2} / \mathrm{cm}^{2}$. The yield is always given as a function of initial thickness, since the film thickness and, therefore, the yield change substantially during the experimental run. A clear indication of the saturation is shown in Fig. 3(a); the erosion rate is constant during the run for the thickest films.

A crucial point is the different behavior of the ion- and electron-induced yields for thick films. A straightforward explanation is that the accumulated primary ions embedded in the deuterium are neutralized by free electrons from the silver substrate as soon as the film charges up above a certain threshold. Since there are no available mobile positive charges from the metal, the incident electrons generate a space charge in the film. This charge-up occurs at the thicknesses for which the electrons after the slowing down no longer can migrate through the film to the substrate.

From this picture one can describe the behavior of the yields from thin and medium thick films for both types of projectiles. At very thin films the yield is enhanced by beam-induced surface effects. The mechanism has not yet been identified, and the yield enhancement has been observed for other gases and by other groups as well $[11,15,17]$, but the effect at these thin films is unimportant for the present work. Since the flat part of the curve for the yield induced by hydrogen ions represents the "bulk" value of the electronic sputtering 


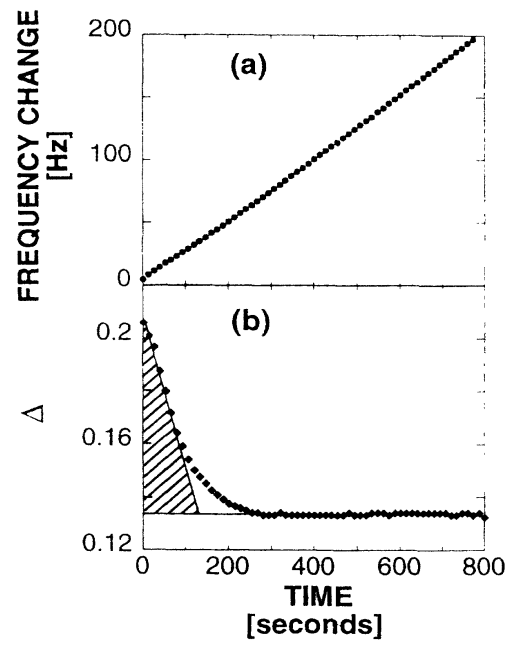

FIG. 3. Erosion curves for a thick film as a function of time: (a) Frequency change for a $11 \times 10^{18} \mathrm{D}_{2} / \mathrm{cm}^{2}$ thick film. (b) The relative current change $\Delta\left(\Delta I_{B}=I_{B}-I_{T}\right)$ for a $7.2 \times 10^{18} \mathrm{D}_{2} / \mathrm{cm}^{2}$ thick film; the dashed area corresponds to the accumulated electron charge.

yield, one may consider the thickness range between $1.0 \times 10^{18}$ and $2.0 \times 10^{18} \mathrm{D}_{2} / \mathrm{cm}^{2}$ with the minimum value of the electron-induced sputtering yield as a bulk region. This idea is corroborated by the fact that the electronic sputtering yield predicted on the basis of lowenergy linear collision cascade theory $[11,18]$ agrees well with the yield in this region.

Obviously, the very large yield for the thick films cannot be explained by any simple electronic sputtering mechanism as the region with the minimum yield. This is supported by our measurements of the target current $I_{T}$ during the initial phase of the erosion. $\Delta$ decreases towards a saturation value, but has a pronounced "shorttime" component around $100 \mathrm{~s}$. Usually, the current difference $\Delta I_{B}$ accounts for the reflected as well as the trapped electrons in the solid [19]. Therefore, the area in Fig. 3(b) corresponds to the number of electrons that do not reach the electrode; i.e., they are trapped in the solid or reflected. In the following we shall denote this number as accumulated charge, even though some of the primary and secondary electrons may be backscattered in addition to the ordinary number of reflected electrons from a film without excess charge. The accumulated charge as a function of film thickness is shown in Fig. 1(c). The charge increases with thickness practically as a linear function with a threshold close to the thickness for which the strong yield enhancement occurs.

One may extract the accumulated excess electron density from the slope in Fig. 1(c) [20]. This slope corresponds to a density of about $7 \times 10^{17}$ electrons $/ \mathrm{cm}^{3}$ which is about a factor of 7 larger than the density of the moderately mobile polaronic electrons and almost 2 orders of magnitude larger than that of the electron bub- bles measured by Brooks and co-workers [9,10]. This discrepancy may be at least partly explained by different bombardment conditions, because the primary ions leave the macroscopically neutral deuterium sample in Brooks's experiment.

The steady-state erosion for thick films, which occurs on a time scale of less than a second, may be described qualitatively by the following scenario: Initially the distribution of excess electrons peaks around the projected electron range, but with increasing fluence some electrons migrate to the metal substrate as a result of the field generated by the other electrons. Eventually, about 0.9 electron per incident electron on the average reaches the substrate, whereas 0.13 electron per incident one is reflected from the film into vacuum. The energy release for sputtering is caused by slow electrons that are driven towards the surface, e.g., the primary electron after slowing down, fast secondaries, or migrating polaronic electrons. Such an electron drift towards the surface is supported by the fact that the thick-film yield for $2 \mathrm{keV}$ electron bombardment approaches $1000 \mathrm{D}_{2}$ /electron in contrast to ordinary electronic sputtering that would lead to a decreasing yield with increasing primary energy $[2,5,15]$.

The energy conversion from kinetic energy of the incident electrons to translational energy of the $D_{2}$ molecules is not identified, but it must be induced by a mechanism entirely different from that of the minimum region. Even conceivable conversion processes by these low-energy electrons such as excitations up to the repulsive $b^{3} \Sigma_{u}^{+}$or to vibrationally excited levels with subsequent relaxation cannot account for the high yields for the thick films. Another possibility is bubble formation in defects close to the surface. Since films produced on a cold substrate have a high defect density [21], electron trapping at defects close to the surface may occur and this bubble formation may release up to $4 \mathrm{eV}[8,10]$ to the surrounding molecules. The localization of the polaronic electrons may also liberate energy. The magnitude of this release is not known, but it is probably more than 1 order of magnitude less than the bubble energy.

In summary, we have presented the first sputtering measurements of thick deuterium films by keV electrons. The sputtering yield for $1.5 \mathrm{keV}$ electrons increases around $5 \times 10^{18} \mathrm{D}_{2} / \mathrm{cm}^{2}(1.6 \mu \mathrm{m})$ by 2 orders of magnitude up to a plateau about $350 \mathrm{D}_{2}$ /electron. A similar behavior at film thicknesses so large has not been observed for any other solidified gas. This enhancement is probably caused by an accumulation of projectile electrons in terms of trapping in bubbles or formation of polarons. The density of electrons in the thick films is estimated to $7 \times 10^{17}$ electrons $/ \mathrm{cm}^{3}$. No existing model can account satisfactorily for the sputtering mechanism as well as the influence of the stored electron charge of the thick films.

The authors thank R.L. Brooks and P. Børgesen for valuable discussions and appreciate the competent 
technical assistance from Arne Nordskov. This work was supported by a grant from the Danish Natural Science Research Council.

Part of this work was performed in the Department of Solid State Physics.

*Present address: Odense University Library, Odense University, DK-5230 Odense M, Denmark.

[1] B. Stenum, J. Schou, O. Ellegaard, H. Sørensen, and R. Pedrys, Phys. Rev. Lett. 67, 2824 (1991).

[2] R. E. Johnson and J. Schou, Mat. Fys. Medd. K. Dan. Vidensk. Selsk. 43, 403 (1993).

[3] I. F. Silvera, Rev. Mod. Phys. 52, 393 (1980).

[4] P.C. Souers, Hydrogen Properties for Fusion Energy (Univ. of California Press, Berkeley, 1986).

[5] J. Schou, Nucl. Instrum. Methods Phys. Res., Sect. B 27, 188 (1987).

[6] J. Schou, H. Sørensen, and P. Børgesen, Nucl. Instrum. Methods Phys. Res., Sect. B 5, 44 (1984).

[7] P. G. LeComber, J. B. Wilson, and R. Loveland, Solid State Commum. 18, 377 (1976).

[8] S. K. Bose and J. D. Poll, Can. J. Phys. 63, 94 (1985); 63, 1105 (1985).

[9] R. L. Brooks, S. K. Bose, J. L. Hunt, J. R. McDonald, J. D. Poll, and J. C. Waddington, Phys. Rev. B 32, 2478 (1985); R. L. Brooks, M. A. Selen, J. L. Hunt, J.R. McDonald, J. D. Poll, and J. C. Waddington, Phys. Rev. Lett. 51, 1077
(1983).

[10] J. J. Miller, R. L. Brooks, and J. L. Hunt, Can. J. Phys. 71, 501 (1993); R. L. Brooks and J. L. Hunt, Phys. Can. 47, 132 (1991).

[11] J. Schou, Ph.D. thesis, Risø National Laboratory [Risø-R591-(EN), 1991].

[12] B. Stenum, O. Ellegaard, J. Schou, and H. Sørensen, Nucl. Instrum. Methods Phys. Res., Sect. B 48, 530 (1990).

[13] P. Børgesen and H. Sørensen, Phys. Lett. 90A, 319 (1982).

[14] O. Ellegaard, Ph.D. thesis, Risø National Laboratory [Risø Rep. M-2617, 1986].

[15] J. Schou, P. Børgesen, O. Ellegaard, H. Sørensen, and C. Claussen, Phys. Rev. B 34, 93 (1986).

[16] S. Valkealahti, J. Schou, H. Sørensen, and R. Nieminen, Nucl. Instrum. Methods Phys. Res., Sect. B 34, 321 (1988).

[17] S. K. Erents and G. M. McCracken, J. Appl. Phys. 44, 3139 (1973).

[18] O. Ellegaard, J. Schou, H. Sørensen, and P. Børgesen, Surf. Sci. 167, 474 (1986).

[19] J. Cazaux, in Ionization of Solids by Heavy Particles, edited by R. Baragiola (Plenum, New York, 1993), p. 325.

[20] B. Thestrup, W. Svendsen, J. Schou, and O. Ellegaard (to be published).

[21] G. Zimmerer, in Excited-State Spectroscopy in Solids, edited by U.M. Grassano and N. Terzi (North-Holland, Amsterdam, 1987), p. 37. 


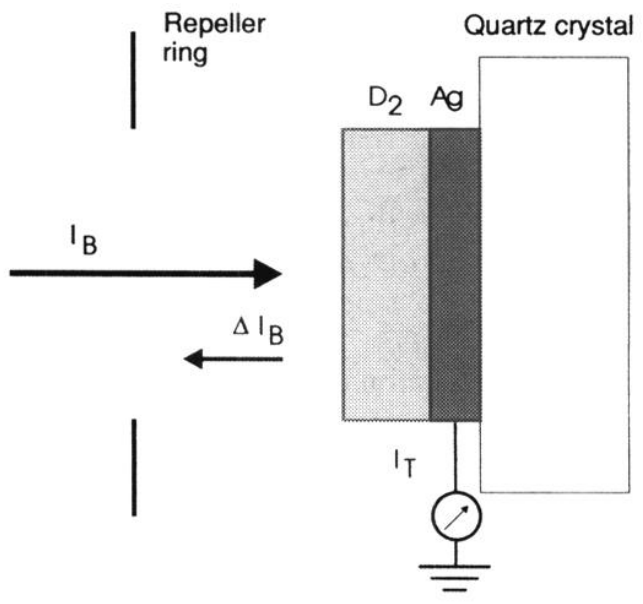

FIG. 2. Schematic view of the target area. The currents are shown as well. 